\title{
Plasma indoleamine 2,3-dioxygenase activity as a potential biomarker for early diagnosis of multidrug-resistant tuberculosis in tuberculosis patients
}

This article was published in the following Dove Press journal:

Infection and Drug Resistance

\author{
Wen Shi ${ }^{1}, *$ \\ Juan $\mathrm{Wu}^{\mathrm{I}, *}$ \\ Qi $\operatorname{Tan}^{\prime}$ \\ Chun-Mei Hu${ }^{2}$ \\ Xia Zhang ${ }^{2}$ \\ Hong-Qiu Pan ${ }^{3}$ \\ Zhen Yang ${ }^{4}$ \\ Meng-Yu $\mathrm{He}^{\prime}$ \\ Min Yu' \\ Bo Zhang ${ }^{5}$ \\ Wei-Ping Xie' \\ Hong Wang' \\ 'Department of Respiratory and Critical \\ Care Medicine, Jiangsu Province Hospital. \\ The First Affiliated Hospital of Nanjing \\ Medical University, Nanjing, Jiangsu \\ Province, People's Republic of China; \\ ${ }^{2}$ Department of Tuberculosis, The Second \\ Hospital of Nanjing, Nanjing, Jiangsu \\ Province, People's Republic of China; \\ ${ }^{3}$ Department of Tuberculosis, The Third \\ Hospital of Zhenjiang City, Zhenjiang, \\ Jiangsu Province, People's Republic of China; \\ ${ }^{4}$ Department of Respiratory Medicine, Jiangbei \\ Hospital, Nanjing, Jiangsu Province, People's \\ Republic of China; ${ }^{5}$ Department of Population \\ and Quantitative Health Sciences, University of \\ Massachusetts Medical School, Worcester, MA, \\ USA
}

*These authors contributed equally to this work

Correspondence: Qi Tan; Wei-Ping Xie Department of Respiratory and Critical Care Medicine, Jiangsu Province Hospital,

The First Affiliated Hospital of Nanjing Medical University, 300 Guangzhou Rd,

Nanjing 210029, Jiangsu Province, People's Republic of China

Tel + I 508330 6097; +86 I38 5I50 68I8

Email qi.tan@umassmed.edu;

wpxie@njmuedu.cn
Purpose: Multidrug-resistant tuberculosis (MDR-TB) remains a challenge of global TB control, with difficulty in early detection of drug-sensitive tuberculosis (DS-TB). We investigate the diagnostic significance of IDO as a potential biomarker to discriminate MDR patients among the TB patients.

Patients and methods: Plasma indoleamine 2,3-dioxygenase (IDO) was measured by the ratio of kynurenine (Kyn) to tryptophan (Trp) concentrations, using high performance liquid chromatography-mass spectrometry (LC-MS/MS). Chest computed tomography (CT) imaging signs from TB patients were collected and analyzed in 18 DS-TB patients, 16 MDR-TB patients, 6 lung cancer (LC) patients, and 11 healthy individuals. Lung imaging signs from TB patients were collected and analyzed.

Results: We found that plasma IDO activity was significantly higher in the MDR-TB patients than in the DS-TB patients $(p=0.012)$ and in the LC patients $(p=0.003)$. We evaluated the diagnostic significance of plasma IDO activity in discriminating the MDRTB group from the DS-TB group using a receiver operating characteristic (ROC) curve. With a cutoff level of $46.58 \mathrm{uM} / \mathrm{mM}$, the diagnostic sensitivity, specificity, positive predictive value (PPV), and negative predictive value (NPV) for IDO activity were $87.50 \%, 72.22 \%$, $73.68 \%$, and $86.67 \%$, respectively. Plasma IDO activity was higher in cavity cases than in non-cavity cases $(p=0.042)$, proving a positive correlation between lung cavity number and cavity size ( $p<0.05$, separately) among all the TB patients studied.

Conclusion: Our findings confirmed that plasma IDO activity might have an auxiliary diagnosis value for early discrimination of MDR-TB patients from DS-TB patients. Among the TB patients with cavitary lung lesions, higher plasma IDO activity can indicate a higher risk of MDR-TB.

Keywords: IDO, MDR-TB, LC-MS/MS, cavitary lung lesion

\section{Introduction}

Multidrug-resistant tuberculosis (MDR-TB) is a major threat to global tuberculosis control. ${ }^{1}$ In 2017, there were 10 million new and relapse tuberculosis (TB) cases worldwide; over 558,000 of these TB patients suffered from MDR-TB, and only 25\% of MDRTB patients were notified. ${ }^{2}$ Missing people with MDR-TB, due to a failure of the local healthcare system ${ }^{3}$ and lack of tools for diagnosis ${ }^{4}$ can lead to the systematic selection and spread of MDR-TB strains. This increases the risk that we may lose control of TB in 
the future. ${ }^{5}$ In comparison with drug-sensitive tuberculosis (DS-TB), MDR-TB treatments tend to use more toxic secondline anti-TB drugs in combination, and in a prolonged treatment regimen (the standard is 9-24 months for MDR-TB versus 6 months for DS-TB) ${ }^{6}$ with less treatment efficiency, potential patient incompliance and, unaffordably high treatment costs. ${ }^{7,8}$ Clinical confirmation of MDR-TB mostly depends on identification of Mycobacterium tuberculosis (MTB) by smear or culture and determination of the strain by drug-susceptibility testing (DST). However, this testing usually takes longer than four weeks. ${ }^{9,10}$ More efficient methods based on GeneXpert/RIF have been recommended by the World Health Organization (WHO) for screening drug resistant-TB. ${ }^{11}$ However, these methods are limited in early detection for MDR-TB due to inadequate sensitivity (46\%), and giving a false-positive result when few bacilli are present in a clinical specimen (smear negative). ${ }^{12,13}$ Current determination of TB in immunology, pathology, and radiology cannot discriminate drug-resistant TB from drug-sensitive TB. ${ }^{14,15}$ The lack of adequate tools is contributing to delayed detection or even missed diagnoses of MDR-TB. ${ }^{16}$ As a result, these missed cases can transmit the pathogen, and extend the MDRTB population in uncontrolled conditions.

Indoleamine 2,3-dioxygenase (IDO) is an intracellular, non-secreted enzyme, and is a key rate-limiting enzyme that catalyzes the catabolism of tryptophan (Trp) to kynurenine (Kyn). ${ }^{17,18}$ Induction of IDO in cells of the immune system by IFN- $\gamma$ is a technique that was developed in the late 1980 s. $^{19,20}$ due to the anti-proliferative features of IDO on bacteria, protozoa, and tumor cells. ${ }^{21}$ Numerous studies in the past two decades have indicated that induced IDO acts as a suppressive regulator in host immunity to promote and prolong TB infection. ${ }^{12-26}$ In both animal models and patient specimens, IDO was found to be enriched in the inner layer of the granuloma ${ }^{22,23}$ and capable of promoting long-term survival of the bacilli by limiting the proliferation of $\mathrm{CD}^{+} \mathrm{T}$ cells and macrophages. ${ }^{27}$ Recent studies have demonstrated that IDO activity in the serum, sputum, or pleura from pulmonary TB (PTB) patients is increased, and can be an independent predictor of mortality. ${ }^{24,26}$ Most of these studies were focused on TB patients; yet, these studies did not distinguish whether the TB patients infected with drugsensitive or drug-resistant MTB strain. So, we still cannot determine the role that IDO plays in MDR-TB infection. Therefore, the differences in IDO activity in MDR-TB patients compared to DS-TB patients have not yet been adequately investigated.
In our previous work, MDR-TB patients-mostly with more severe clinical symptoms and radiological lung focuses-were recognized by an extreme drop of $\mathrm{CD} 4^{+} \mathrm{T}$ helper subset cell responses before treatment which was markedly different from that in DS-TB cases, including a more sharply decreased Th1 frequency, lower IFN- $\gamma$ concentration, and higher over-induced inflammatory cytokine expressions in peripheral blood mononuclear cells (PBMCs). ${ }^{28,29}$ Thus, we hypothesize that the increased Trp and decreasing Kyn levels within IDO activity play a crucial role. In this study, we investigate the diagnostic significance of IDO as a potential biomarker to discriminate MDR patients from other TB patients. We also investigated the IDO activity identification significance in MDR-TB patients compared with lung cancer (LC) patients, and the correlation of plasma IDO activity with the frequency and size of cavitary lung lesions of TB patients.

Our findings indicated plasma IDO activity was a novel biomarker for early diagnosis MDR-TB, and suggested a potential target of host direct treatment (HDT) for MDRTB in future by indicating the crucial role of IDO in the immune response against MDR-TB. Our findings confirmed that plasma IDO activity can remarkably discriminate MDR-TB patients from DS-TB patients, as well as from lung cancer patients. Meanwhile we demonstrated a higher plasma IDO activity in cavitary TB patients than in non-cavitary TB patients. Plasma IDO activity level was illustrated a positive correlation with the number and size of lung cavitary lesions in patients with TB, according to imaging signs. This hinted that plasma IDO activity can also be a biomarker to boost imaging signs of the cavity, indicating the risk of MDR-TB infection and pathogen transmittance. In conclusion, our pilot investigation sheds light on plasma IDO activity performance in the early identification of MDR-TB patients, which may be an auxiliary tool for promptly conducting the correct patients into an isolation ward and thereby alleviating the spread of MDR-TB.

\section{Material and methods}

\section{Ethics statement}

This study protocol was approved by the ethics committee of the First Affiliated Hospital of Nanjing Medical University (Jiangsu Province Hospital) and was performed adhering to the ethical principles of the Declaration of Helsinki (APPROVAL NUMBER/ID 2017-SRFA-163). All patients provided written informed consent before enrollment. 


\section{Subjects and diagnostic criteria}

From December 2017 to May 2018, 34 newly diagnosed pulmonary TB patients, including 18 cases of DS-TB, 16 cases of MDR-TB, and 6 cases of LC, along with 11 HCs, were enrolled in the study and admitted to The Public Health Medical Center of Nanjing City, Jiangbei Hospital, Third Hospital of Zhenjiang City, and The First Affiliated Hospital of Nanjing Medical University. All participants were over 18 years old. All of the active tuberculosis patients recruited were bacteriologically confirmed, and presented with a protracted cough, occasional fever, blood-streaked phlegm or hemoptysis, night sweats, fatigue, weight loss, and a positive chest computed tomography (CT) with lung lesions and/or cavities. TB patients with human immunodeficiency virus (HIV), cancer, autoimmune diseases, liver and kidney diseases, undergoing immunosuppressive treatment, blood system dysfunction, or who were pregnant or lactating women were excluded. DS-TB patients were confirmed by a positive bacterial culture with drug-sensitive stains and had no history of TB. MDR-TB patients were diagnosed with a positive sputum culture and baseline drug sensitivity tests using at least two drugs-isoniazid and rifampin. LC patients were diagnosed on the basis of detecting malignant cells in a pulmonary biopsy. Our study contemporaneously enrolled healthy controls (HCs) with normal chest $\mathrm{X}$-rays, no cough, fever, or weight loss, and no recent exposure to TB.

\section{Determination of radiological findings}

Of the 34 pulmonary TB patients, chest $\mathrm{CT}$ scans showing the lung lesions of 18 patients with DS-TB and 16 patients with MDR-TB were collected before initial treatment regimen and reviewed in a random order by a medical group of senior physicians in the department of respiratory medicine. The determination of the imaging signs included the lung cavity prevalence, bilateral lungs, cavity number, maximum cavity diameter, and sum diameters of all cavities. Radiological findings on the chest CT scans of individual patients were confirmed by the physician's decision.

\section{Measurement of tryptophan and kynurenine Chemicals}

L-Trp (Sigma-Aldrich, USA) and L-Kyn (Sigma-Aldrich, USA) were dissolved in methanol and stored frozen at $20{ }^{\circ} \mathrm{C}$. Methanol and formic acid (high-performance liquid chromatography (HPLC) grade) were purchased from Tedia (USA). Other organic solvents and chemicals were all HPLC grade.

\section{Analysis parameters of liquid chromatography-tandem mass spectrometry (LC-MS/MS)}

The solvent and sample were separated by an auto sampler (Agilent, USA) and a chromatographic pump (Agilent, USA). The column was Reversal Phase-18 (RP-18) with C18 protection pre-column. The column oven was set at $25{ }^{\circ} \mathrm{C}$. The mobile phase was methanol/water $(95: 5 \mathrm{v} / \mathrm{v})$ (water containing $0.1 \%$ formic acid), and the flow rate of the LC system was $200 \mu \mathrm{l} / \mathrm{min}$. The analysis time for each serum sample was four minutes.

The sample profiling analysis was performed by a triple quadrupole mass spectrometer QTRAP ${ }^{\circledR} 5000$ (AbSciex, USA) with an ion source of electrospray ionization (ESI); a detection method of positive ion detection; a detection molecular weight of $209.1 \rightarrow 145.9$ (Kyn) and $205.3 \rightarrow 149.0$ (Trp); a scanning method of selection reaction monitoring; a scanning time of $0.1 \mathrm{~s}$; a scanning range of $10-300 \mathrm{~m} / \mathrm{z}$ (Kyn) and 10-300 m/z (Trp); and a collision energy of $12.7 \mathrm{~V}$ (Kyn) and $22.3 \mathrm{~V}$ (Trp).

\section{Sample and plasma pretreatment}

The stock solutions of L-Kyn and L-Trp were further diluted with methanol into a series of working solutions and stored at $-20{ }^{\circ} \mathrm{C}$, and were thawed at room temperature before $\mathrm{LC} /$ MS analysis. The standard sample concentration ranges were $10-500 \mathrm{ng} / \mathrm{ml}$ (Kyn) and 12.5-400 ng/ml (Trp).

Ethylenediaminetetraacetic acid-anticoagulated plasma samples were obtained according to the standard operating procedure at the time of admission, and $100 \mu \mathrm{l}$ of plasma and $300 \mu \mathrm{l}$ of methanol were added to a $1.5 \mathrm{ml}$ centrifuge tube. These were centrifuged at $12,000 \mathrm{rpm}$ for $30 \mathrm{~min}$ at $4{ }^{\circ} \mathrm{C}$. Then, the supernatants were collected and stored at $80{ }^{\circ} \mathrm{C}$. After this, the mixture was acutely vortexed for $10 \mathrm{~s}$ and centrifuged at $12,000 \mathrm{rpm}$ for $5 \mathrm{~min}$ at $4{ }^{\circ} \mathrm{C}$, then the supernatant was transferred to the auto sampler. The injection volume of every sample was $10 \mu 1$ for LC-MS/MS.

The plasma IDO activity was estimated by the plasma ratio of the Kyn concentration to the Trp concentration.

\section{Statistical analysis}

Summary statistics were shown for the continuous measurements with their means and standard deviations (SDs), and for the categorical measurements with their 
frequencies and percentages. Logarithmic transformations were performed for the Kyn concentrations and IDO activities to avoid the influence of non-normality. Transformed Kyn concentrations and IDO activities were adopted in the statistical analysis. A Student $t$-test was used to compare each of the two groups. A one-way analysis of variance (ANOVA) test with the least significant differences (LDSs) post-hoc test was used for multiple-group comparison. Categorical data were analyzed using Fisher's exact tests. The Mann-Whitney test was used for nonparametric unpaired groups. The diagnostic significance of the IDO activity in discriminating the MDR-TB patients from the DS-TB and LC patients was evaluated by an area under the receiver operating characteristic (ROC) curve (AUC), sensitivity, specificity, positive predictive value (PPV), and negative predictive value (NPV). Spearman's $t$-test was used to detect the correlation between the Try-Kyn pathway and radiological findings. Data analysis were performed using SPSS software (SPSS 25.0, Chicago, IL, USA), and a significance level of 0.05 was considered in the hypothesis testing.

\section{Results}

\section{Demographics and clinical parameters}

A total of 51 participants were included, with $11 \mathrm{HCs}$, 18 DS-TB cases, 16 MDR-TB cases, and 6 LC cases (3 lung adenocarcinoma cases, 2 squamous cell lung cancer cases, and 1 small cell lung cancer case). The baseline demographic characteristics of the study participants are summarized in Table 1. There was no difference among groups in the distribution of age, gender, body mass index (BMI), or smoking status. Meanwhile, radiological findings of the DS-TB and the MDR-TB patients are summarized in Table 2. A higher proportion of the MDR-TB patients demonstrated cavitary lung lesions compared to the DS-TB patients. No difference of bilateral lung lesion prevalence between the DS-TB patients and the MDRTB patients was found. Figure 1 shows the imaging signs of the DS-TB (Figure 1A and B) and MDR-TB patients (Figure 1C and D).

\section{Plasma concentrations of Kyn, Trp, and IDO activity in the four study groups}

As shown in Figure 2A, the Kyn concentration in plasma was significantly higher in patients with MDR-TB than in the HC, DS-TB, and LC groups $(988.26 \pm 332.75 \mathrm{nM}$ vs $757.33 \pm 160.98 \mathrm{nM}, p=0.013 ; 988.26 \pm 332.75 \mathrm{nM}$ vs $743.50 \pm 145.42 \mathrm{nM}, p=0.003 ; 988.26 \pm 332.75 \mathrm{nM}$ vs $725.79 \pm 103.42 \mathrm{nM}, p=0.022$ ), whereas no significant difference was found between the HC and DS-TB groups $(757.33 \pm 160.98 \mathrm{nM}$ vs $743.50 \pm 145.42 \mathrm{nM}, p=0.844)$ (Figure 2A). Compared with MDR-TB patients, the HC, DS-TB, and LC patients had a significantly higher Trp concentration $\quad(23.21 \pm 5.35 \mu \mathrm{M} \quad$ vs $12.19 \pm 3.79 \mu \mathrm{M}$, $p<0.0001 ; 19.05 \pm 6.11 \mu \mathrm{M}$ vs $12.19 \pm 3.79 \mu \mathrm{M}, p<0.0001$; $21.27 \pm 5.65 \mu \mathrm{M}$ vs $12.19 \pm 3.79 \mu \mathrm{M}, p=0.001$ ) (Figure $2 \mathrm{~B}$ ). In addition, we also observed a decreasing trend in the plasma Trp concentration in the DS-TB group compared with the $\mathrm{HC}$ group, with the difference being significant (19.05 $\pm 6.11 \mu \mathrm{M}$ vs $23.21 \pm 5.35 \mu \mathrm{M}, p=0.044)$. As a result, our data revealed significantly higher IDO activity (Kyn/ Trp ratio) in the MDR-TB individuals than in the HC, DS$\mathrm{TB}$, and LC groups $(90.61 \pm 49.09 \mu \mathrm{M} / \mathrm{mM}$ vs 35.18 $\pm 13.92 \mu \mathrm{M} / \mathrm{mM}, p=0.003 ; 90.61 \pm 49.09 \mu \mathrm{M} / \mathrm{mM}$ vs 43.84 $\pm 19.53 \mu \mathrm{M} / \mathrm{mM}, p=0.012 ; 90.61 \pm 49.09 \mu \mathrm{M} / \mathrm{mM}$ vs 35.91 $\pm 9.56 \mu \mathrm{M} / \mathrm{mM}, p=0.003$ ), whereas no significant difference was found between the HC and DS-TB groups (35.18 $\pm 13.92 \mu \mathrm{M} / \mathrm{mM}$ vs $43.84 \pm 19.53 \mu \mathrm{M} / \mathrm{mM}, \quad p=0.687)$ (Figure 2C).

Table I Baseline characteristics of the participants in the four groups

\begin{tabular}{|c|c|c|c|c|c|}
\hline Variable & $\begin{array}{l}\text { HC } \\
(n=I I)\end{array}$ & $\begin{array}{l}\text { DS-TB } \\
(n=18)\end{array}$ & $\begin{array}{l}\text { MDR-TB } \\
(n=16)\end{array}$ & $\begin{array}{l}\text { LC } \\
(n=6)\end{array}$ & p-value \\
\hline Age (years) & $51.09 \pm 10.77$ & $48.72 \pm 15.97$ & $58.00 \pm 6.67$ & $60.50 \pm 3.73$ & $0.053^{\mathrm{a}}$ \\
\hline Gender (male/female) & $8 / 3$ & $14 / 4$ & $13 / 3$ & $5 / 1$ & $0.946^{\mathrm{b}}$ \\
\hline BMI $\left(\mathrm{kg} / \mathrm{m}^{2}\right)$ & $21.11 \pm 1.40$ & $20.56 \pm 3.24$ & $20.73 \pm 2.20$ & $22.48 \pm 2.54$ & $0.437^{\mathrm{a}}$ \\
\hline Smoking (no/yes) & $8 / 3$ & $7 / 11$ & $6 / 10$ & $1 / 5$ & $0.113^{b}$ \\
\hline Type of clinical TB & Non-TB & Pulmonary TB & & Non-TB & \\
\hline
\end{tabular}

Notes: ${ }^{a}$ One-way analysis of variance (ANOVA) test was used for the multiple-group comparison. ${ }^{b}$ Fisher exact test was used for categorical data. Data are shown as mean \pm SD or ratios.

Abbreviations: HC, healthy control; DS-TB, drug-sensitive tuberculosis; MDR-TB, multidrug-resistant tuberculosis; LC, lung cancer; BMI, body mass index. 
Table 2 Radiological characteristics of the DS-TB and MDR-TB patients

\begin{tabular}{|l|l|l|l|}
\hline Radiological findings & $\begin{array}{l}\text { DS-TB } \\
(\mathbf{n = 1 8 )}\end{array}$ & $\begin{array}{l}\text { MDR-TB } \\
(\mathbf{n}=16)\end{array}$ & $\boldsymbol{P}$-value \\
\hline Cavity prevalence [n(\%)] & $6(33.33 \%)$ & $13(81.25 \%)$ & $0.007^{* \mathrm{a}}$ \\
Cavity number $(\mathrm{n})$ & $0.50 \pm 0.79$ & $2.44 \pm 2.25$ & $0.00 \mathrm{I}^{\mathrm{b}}$ \\
Bilateral lung lesions [n(\%)] & $2(11.11 \%)$ & $5(31.25 \%)$ & $0.214^{\mathrm{c}}$ \\
Maximum diameter of cavity (mm) & $8.33 \pm 13.39$ & $22.19 \pm 18.79$ & $0.012^{*} \mathrm{~b}$ \\
Sum of cavity diameters (mm) & $10.56 \pm 16.97$ & $34.38 \pm 29.26$ & $0.006^{*} \mathrm{~b}$ \\
\hline
\end{tabular}

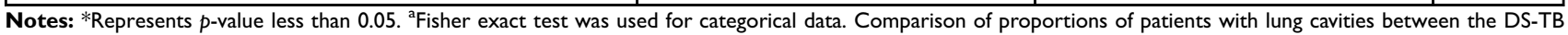
group and MDR-TB group. ${ }^{b}$ Mann-Whitney test was used for nonparametric unpaired groups. ${ }^{c}$ Fisher exact test was used for categorical data. Comparison of proportions of patients with bilateral lung lesions between the DS-TB group and MDR-TB group. Data are shown as mean \pm SD or percentages.

Abbreviations: DS-TB, drug-sensitive tuberculosis; MDR-TB, multidrug-resistant tuberculosis.

\section{Diagnostic significance of plasma concentrations of Kyn, Trp, and IDO activity for discriminating MDR-TB patients from TB patients}

To evaluate the potential values of the plasma tryptophankynurenine pathway for the diagnosis of MDR-TB, three ROC curves were plotted (Figure 3). Using optimal cut-off points, the diagnostic ability of these parameters is shown in
Table 3. At a cutoff of $46.58 \mu \mathrm{M} / \mathrm{mM}$, IDO activity had a sensitivity of $87.50 \%$, a specificity of $72.22 \%$, a PPV of $73.68 \%$, and an NPV of $86.67 \%$ (AUC $=0.882$, Figure $3 \mathrm{C}$, Table 3). We also analyzed the diagnostic value of plasma Kyn concentration and Trp concentration, and the two key indicators of the plasma tryptophan-kynurenine pathway can distinguish MDR-TB groups as well (Kyn, AUC $=0.764$; Trp, AUC $=0.833$, Figure $3 \mathrm{~A}$ and $\mathrm{B}$, Table 3 ). In the analysis, plasma IDO activity had a higher AUC than Kyn or Trp concentration.

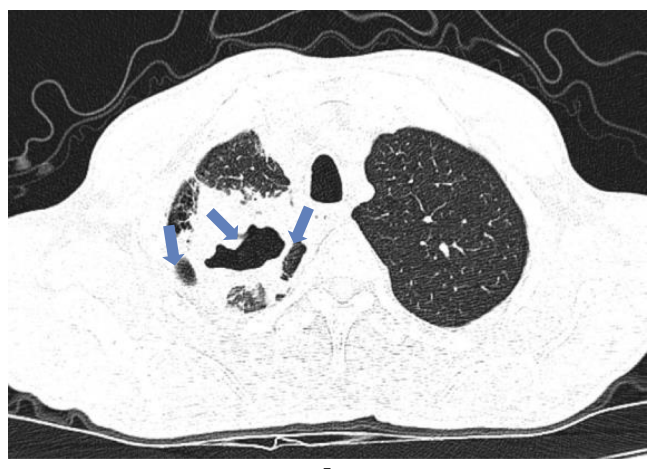

A

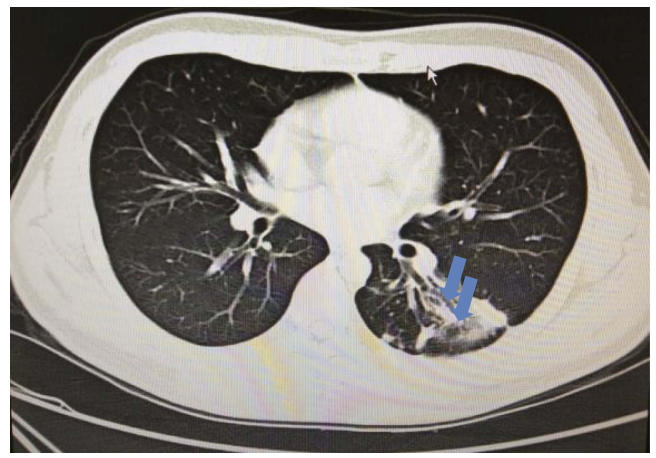

C

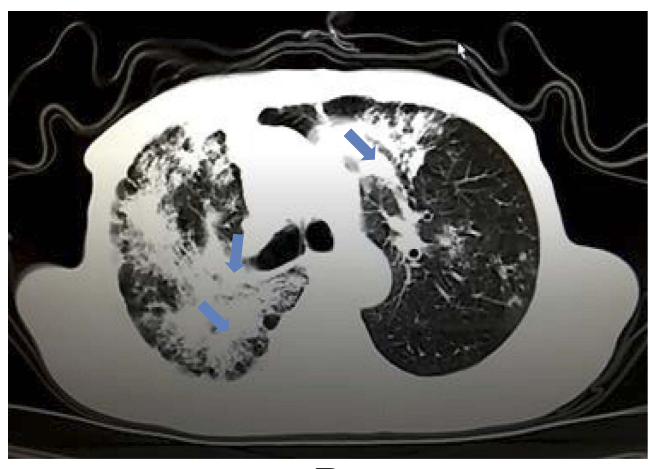

B

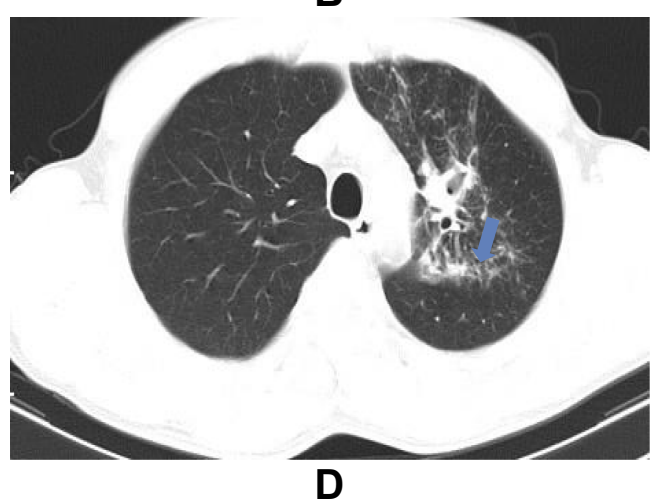

Figure I Imaging signs of transvers CT scans from patients in the drug-sensitive tuberculosis (DS-TB) group and multidrug-resistant tuberculosis (MDR-TB) group. (A) Lung window of chest CT scan from a patient in the MDR-TB group in the present study, showing multiple large cavities (arrows), mainly in the left upper lobe, with lung volume loss. (B) Lung window of chest CT scan from another patient in the MDR-TB group in the present study, showing extensive cavitary lesions (arrows) evolved in the bilateral lungs. (C) Lung window of chest CT scan from a patient in the DS-TB group in the present study, showing at the level of the left basal trunk small nodules branching in the linear and lobular area of bronchiectasis (arrows), with a single cavity formation in the left lower lobe. (D) Lung window of chest CT scan from another patient in the DS-TB group in the present study, showing at the level of the aortic arch nodular opacities and consolidations (arrow), and the lobular area of infiltration lesions. 

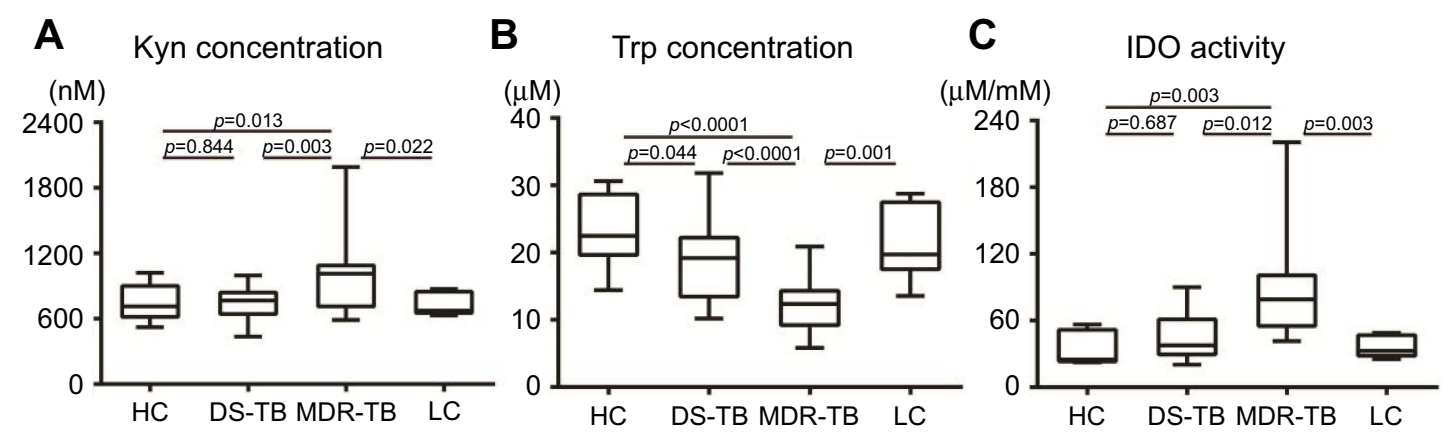

Figure 2 Boxlots of plasma concentrations of Kyn, Trp, and IDO activity in HCs and patients with DS-TB, MDR-TB, and LC. (A) The Kyn concentration in the MDR-TB group was higher than in the HC, DS-TB, and LC groups (MDR-TB vs HC, $p=0.013$; MDR-TB vs DS-TB, $p=0.003$; MDR-TB vs LC, $p=0.022$ ). Meanwhile, no significant difference was found between the $\mathrm{HC}$ and DS-TB groups $(p=0.844)$. (B) The Trp concentration in the MDR-TB group was lower than in the HC, DSTB, and LC groups (MDR-TB vs HC, $p<0.000$ I; MDR-TB vs DS-TB, $p<0.000$ I; MDR-TB vs LC, $p=0.001$ ). In addition, the Trp concentration in the DS-TB group was lower than in the HCs $(p=0.044)$. (C) The IDO activity (Kyn/Trp ratio) in patients with MDR-TB was higher than in the HC, DS-TB, and LC groups (MDR-TB vs HC, $p=0.003$; MDR-TB vs DS-TB, $p=0.012$; MDR-TB vs LC, $p=0.003$ ). Meanwhile, no significant difference was found between the HC and DS-TB groups $(p=0.687)$.

Abbreviations: Kyn, kynurenine; Trp, tryptophan; IDO, indoleamine 2,3-dioxygenase; HC, healthy control; DS-TB, drug-sensitive tuberculosis; MDR-TB, multidrugresistant tuberculosis; LC, lung cancer.

A

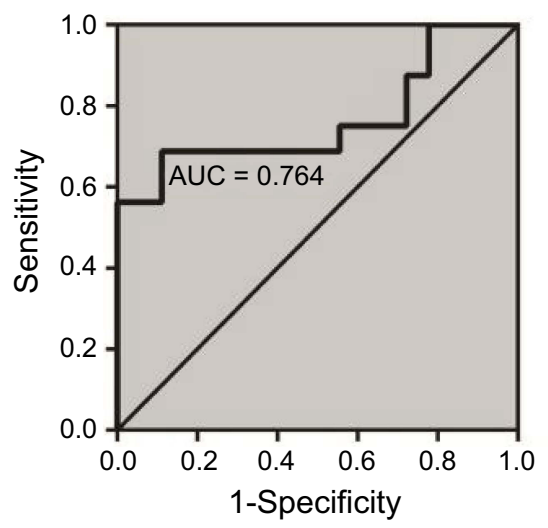

B

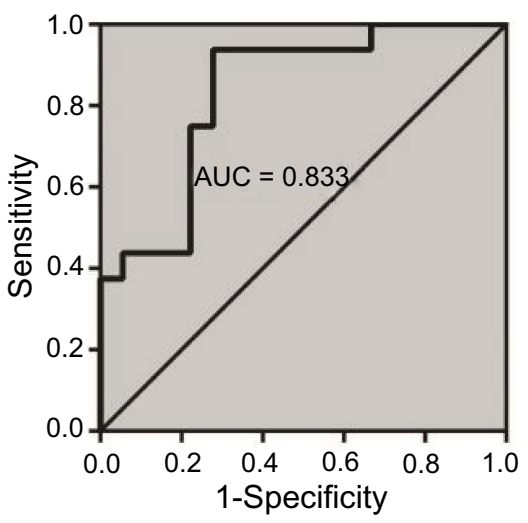

C

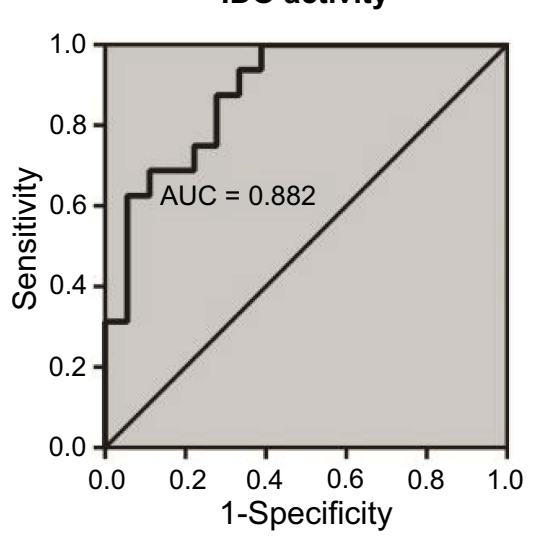

Figure 3 ROC curves of plasma concentrations of Kyn, Trp, and IDO activity for discriminating MDR-TB patients from DS-TB patients. (A) Using a cutoff of 857.5 nM, plasma Kyn concentration had a diagnostic sensitivity of $68.75 \%$ and a specificity of $88.89 \%$ (AUC $=0.764$ ). (B) The AUC was 0.833 for Trp concentration (cutoff value 17.58 $\mu \mathrm{M} / \mathrm{mM}$, sensitivity $93.75 \%$, specificity $72.22 \%$ ). (C) The AUC of IDO activity in differentiating patients with MDR-TB from DS-TB was 0.882 , and the cutoff value was 46.58 $\mu \mathrm{M} / \mathrm{mM}$. Sensitivity and specificity were $87.50 \%$ and $72.22 \%$, respectively.

Abbreviations: Kyn, kynurenine; Trp, tryptophan; IDO, indoleamine 2,3-dioxygenase; AUC, area under the curve; ROC, receiver operating characteristic; MDR-TB, multidrug-resistant tuberculosis; DS-TB, drug-sensitive tuberculosis.

Table 3 Diagnostic significance of plasma concentrations of Kyn, Trp, and IDO activity for discriminating MDR-TB patients from TB patients

\begin{tabular}{|l|l|l|l|l|l|l|}
\hline Variable & AUC & Cutoff & Sensitivity & Specificity & PPV & NPV \\
\hline \multicolumn{7}{|l|}{ DS-TB vs MDR-TB } \\
\hline Kyn & 0.764 & $857.5 \mathrm{nM}$ & $68.75 \%$ & $88.89 \%$ & $84.62 \%$ & $76.19 \%$ \\
Trp & 0.833 & $17.58 \mu \mathrm{M}$ & $93.75 \%$ & $72.22 \%$ & $75.00 \%$ & $92.86 \%$ \\
IDO activity & 0.882 & $46.58 \mu \mathrm{M} / \mathrm{mM}$ & $87.50 \%$ & $72.22 \%$ & $73.68 \%$ & $86.67 \%$ \\
\hline
\end{tabular}

Note: *Represents a $p$-value less than 0.05 .

Abbreviations: AUC, area under the curve; PPV, positive predictive value; NPV, negative predictive value; DS-TB, drug-sensitive tuberculosis; MDR-TB, multidrug-resistant tuberculosis; Kyn, kynurenine; Trp, tryptophan; IDO, indoleamine 2,3-dioxygenase. 


\section{Association of plasma concentrations of Kyn, Trp, and IDO activity with radiological findings}

In addition, we explored the Spearman correlation analysis of the three plasma indexes with radiological findings in the TB (DS-TB, MDR-TB) patients. As presented in Table 4, Trp concentration was negatively correlated with cavity number, the maximum diameter of the cavity, and the sum of cavity diameters $(r=-0.373, p=0.030 ; r=-0.386, p=0.024$; $r=-0.408, p=0.017)$. IDO activity showed a positive association with the maximum diameter of the cavity, and the sum of cavity diameters $(r=0.441, p=0.009 ; r=0.383, p=0.026$; $r=0.410, p=0.016$ ). Subsequently, subgroup analyses were carried out and no significant differences were found in the DSTB and MDR-TB patients separately.

\section{Plasma concentrations of Kyn, Trp, and IDO activity in cavitary and non-cavitary pulmonary TB patients}

Next, we investigated plasma tryptophan-kynurenine pathway indexes in non-cavitary pulmonary TB patients $(n=15)$ and cavitary pulmonary TB patients $(n=19)$ on CT scanning. No significant difference was found in Kyn concentration between the two subgroups, but there was a higher trend in patients with cavities $(911.09 \pm 336.75 \mathrm{nM}$ vs $792.30 \pm 162.21 \mathrm{nM}, p=0.296$ ) (Figure 4A). Compared with non-cavitary TB patients, cavitary patients had significantly lower Trp concentrations $(13.88 \pm 5.21 \mu \mathrm{M}$ vs 18.28 $\pm 6.55 \mu \mathrm{M}, p=0.037$ ) (Figure 4B) and higher IDO activity $(79.03 \pm 50.71 \mu \mathrm{M} / \mathrm{mM}$ vs $49.15 \pm 23.00 \mu \mathrm{M} / \mathrm{mM}$, $p=0.042$ ) (Figure 4C).

\section{Diagnostic value of plasma concentrations of Kyn, Trp, and IDO activity for discriminating LC and MDR-TB patients}

Finally, based on ROC curves, we evaluated the diagnostic significance of plasma tryptophan-kynurenine pathway indexes for distinguishing MDR-TB from other lung diseases (LC). With an optimal calculated IDO activity threshold of $49.23 \mu \mathrm{M} / \mathrm{mM}$, the AUC was 0.958 , with a sensitivity and specificity of $87.50 \%$ and $100.00 \%$, respectively, and with a PPV and NPV of $100.00 \%$ and $75.00 \%$, respectively

Table 4 Correlation coefficient between cavity number, maximum cavity diameter, sum of cavity diameters, and kynurenine, tryptophan, and IDO activity by patient group

\begin{tabular}{|l|l|l|l|l|l|l|l|l|l|l|l|}
\hline & Kyn & Trp & IDO activity & Kyn & Trp & IDO activity & Kyn & Trp & IDO activity \\
\hline & \multicolumn{2}{|l|}{ TB (DS, MDR) patients } & \multicolumn{2}{l|}{ DS-TB patients } & \multicolumn{2}{l|}{ MDR-TB patients } \\
\hline Cavity number $(r)$ & 0.301 & -0.373 & 0.441 & -0.245 & -0.033 & -0.134 & 0.451 & -0.128 & 0.331 \\
$p$-value & 0.084 & $0.030^{*}$ & $0.009 *$ & 0.328 & 0.895 & 0.597 & 0.08 & 0.636 & 0.210 \\
Maximum cavity diameter $(r)$ & 0.141 & -0.386 & 0.383 & -0.228 & -0.068 & -0.085 & 0.104 & -0.317 & 0.324 \\
p-value & 0.427 & $0.024^{*}$ & $0.026^{*}$ & 0.363 & 0.790 & 0.738 & 0.701 & 0.232 & 0.220 \\
Sum of cavity diameters $(r)$ & 0.183 & -0.408 & 0.410 & -0.235 & -0.062 & -0.095 & 0.245 & -0.393 & 0.435 \\
$p$-value & 0.301 & $0.017^{*}$ & $0.016^{*}$ & 0.347 & 0.807 & 0.707 & 0.36 & 0.132 & 0.093 \\
\hline
\end{tabular}

Notes: *Represents $p$-value less than 0.05 . Data are shown as correlation coefficient $(r) ; r$ and $p$-value by Spearman rank correlation.

Abbreviations: Kyn, kynurenine; Trp, tryptophan; IDO, indoleamine 2,3-dioxygenase.
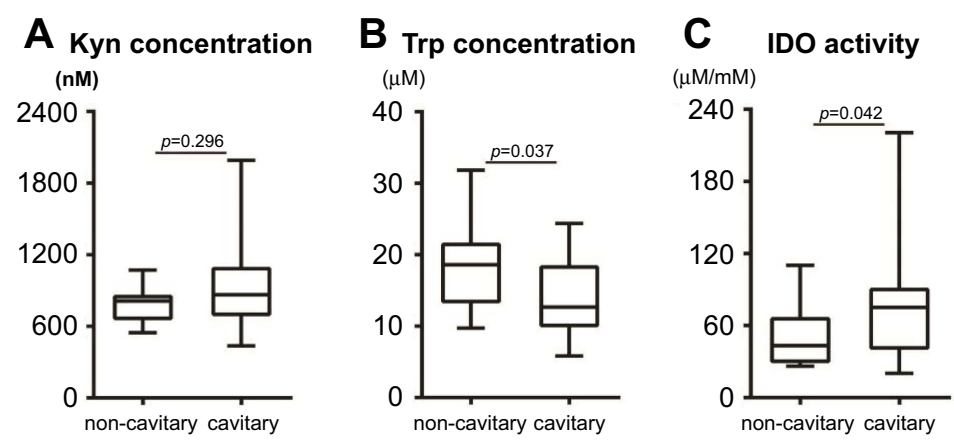

Figure 4 Boxplots of plasma concentrations of Kyn, Trp, and IDO activity in non-cavitary and cavitary TB patients. (A) There was no difference in Kyn concentration between the two groups $(p=0.296)$. (B) The Trp concentration in cavitary TB patients was lower than in non-cavitary TB patients ( $p=0.037)$. (C) The IDO activity in cavitary TB patients was higher than in non-cavitary TB patients $(p=0.042)$.

Abbreviations: Kyn, kynurenine; Trp, tryptophan; IDO, indoleamine 2,3-dioxygenase; TB, tuberculosis. 
A Kyn concentration

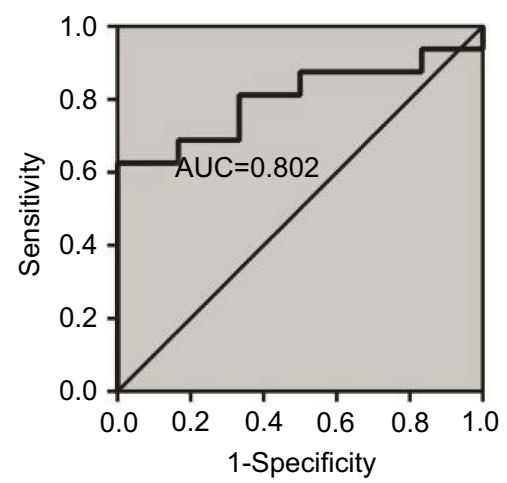

B

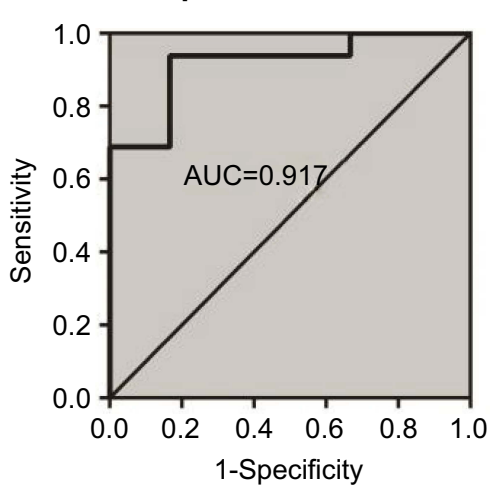

C

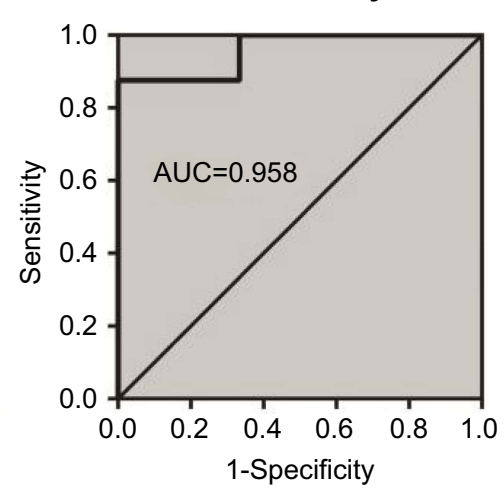

Figure 5 ROC curves of plasma concentrations of Kyn, Trp, and IDO activity for discriminating LC and MDR-TB patients. (A) The AUC of Kyn concentration was 0.802 , with a sensitivity and specificity of $68.75 \%$ and $83.33 \%$, respectively. (B) The sensitivity and specificity of the Trp concentration were found to be $93.75 \%$ and $83.33 \%$, respectively, with an AUC of 0.917 , and a cut-off value of $17.98 \mu \mathrm{M}$ was obtained. (C) The sensitivity and specificity for plasma IDO activity were found to be $87.50 \%$ and $100.00 \%$, respectively (AUC $=0.958)$.

Abbreviations: Kyn, kynurenine; Trp, tryptophan; IDO, indoleamine 2,3-dioxygenase; MDR-TB, multidrug-resistant tuberculosis; DS-TB, drug-sensitive tuberculosis.

Table 5 Diagnostic significance of plasma concentrations of Kyn, Trp, and IDO activity for discriminating MDR-TB patients from the LC patients

\begin{tabular}{|l|l|l|l|l|l|l|}
\hline Variable & AUC & Cutoff & Sensitivity & Specificity & PPV & NPV \\
\hline LC vs MDR-TB & & & & & & \\
Kyn & 0.802 & $852.7 \mathrm{nM}$ & $68.75 \%$ & $83.33 \%$ & $91.67 \%$ & $50.00 \%$ \\
Trp & 0.917 & $17.98 \mu \mathrm{M}$ & $93.75 \%$ & $83.33 \%$ & $93.75 \%$ & $83.33 \%$ \\
IDO activity & 0.958 & 49.23 & $87.50 \%$ & $100.00 \%$ & $100.00 \%$ & $75.00 \%$ \\
\hline
\end{tabular}

Note: *Represents $p$-values less than 0.05 .

Abbreviations: AUC, area under the curve; PPV, positive predictive value; NPV, negative predictive value; MDR-TB, multidrug-resistant tuberculosis; Kyn, kynurenine; Trp, tryptophan; IDO, indoleamine 2,3-dioxygenase; LC, lung cancer.

(Figure 5C, Table 5). The AUCs of Kyn and Trp were 0.802 and 0.917 , respectively (Figure 5A and B, Table 5).

\section{Discussion}

Despite increased research activity in tuberculosis, clinicians remain hindered by gaps in knowledge and understanding of both pathogenesis and the host factors that contribute to the susceptibility of MDR-TB disease. ${ }^{30,31}$ We are clearly lacking host directed biomarkers for indicating disease status, especially in the early stage of MDR-TB. ${ }^{32}$ To date, few studies have discussed IDO activity in MDR-TB patients, either to clearly determine its profile in host immunity against MDR-TB or in lung lesions from MDR-TB patients.

It has been recognized that tryptophan metabolism brings twofold consequences. First, starving the bacteria by depleting the local microenvironment of tryptophan, which is essential for most pathogen metabolism. ${ }^{33,34}$ Second, inducing the apoptosis of host immune cells, ${ }^{35,36}$ mostly lymphocytes activated by tryptophan downstream catabolites, including kynurenine and 3-hydroxyanthranilic acid (3-HAA). IDO activity is evolved in suppressing $\mathrm{CD} 4{ }^{+} \mathrm{T}$ cell proliferation. ${ }^{37}$ inducing antigen-presenting cells (APC). ${ }^{38}$ and promoting the differentiation of $\mathrm{CD}^{+}{ }^{+} \mathrm{CD} 25^{+}{ }^{+}$oxp $^{+}{ }^{+}$regulatory $\mathrm{T}$ cells (Treg) cells from naive $\mathrm{CD}^{+} \mathrm{T}$ cells. ${ }^{37,39,40}$ Therefore, IDO activity is believed to contribute to mycobacteria burden increase and persistence in chronic infection. The present study shows that IDO activity in the MDR-TB group was higher than in the DS-TB group, and IDO activity was also associated with lung cavitary lesions in the patients with TB. This is consistent with our previous finding that MDR-TB patients show lower $\mathrm{CD} 4^{+} \mathrm{IFN}-\gamma^{+} \mathrm{T}$ cell response and overinduced Treg activation in PBMCs along with more severe clinical symptoms and lung lesions. ${ }^{28,29,41}$

Abundant evidence also indicates IDO activity not only participates in the pathogenesis of $\mathrm{TB}$, but also can be a complementary tool for diagnosis and prognosis of TB. ${ }^{23-26,42}$ However, no studies have been conducted to evaluate its diagnostic significance in MDR-TB patients. In this regard, we clearly showed that serum IDO activity 
in MDR-TB discriminates this condition from DS-TB groups, with a cutoff level of $46.58 \mu \mathrm{M} / \mathrm{mM}$, sensitivity $87.50 \%$, specificity $72.22 \%$, and positive predictive value (PPV) of 73.68\%. We also measured plasma Kyn and Trp concentration as some of the IDO metabolic pathway products in MDR-TB patients and consistently found significant differentiations in MDR-TB patients from DS-TB patients. A study in Japan reported in 2011 enrolled 174 consecutive patients and showed that serum IDO activity was an independent predictor of mortality. ${ }^{24}$ A prospective clinical study for TB patients with HIV infection demonstrated that, compared to the higher IDO activity before diagnosis, IDO activity decreased after anti-TB treatment. $^{42}$ A large-scale study will be conducted in future with a well-designed protocol to validate this finding.

In the present study, the IDO activity showed a significant positive correlation with lung cavity prevalence and lung cavity size. We also observed the association of the three plasma indexes with radiological findings in DS-TB and MDR-TB patients separately, and no significant differences were found. We further found IDO activity was relatively higher in 19 patients with pulmonary cavities, compared with 15 patients who presented with non-cavities on computed tomography (CT) scanning. Based on accumulating evidence from studies on animal and human specimens, $M$. tuberculosis infection is associated with high expression of IDO in the infected phagocytes in the center structure of the granuloma, preventing $\mathrm{T}$ cell protection and thereby further promoting bacterial survival, which leads to cavitary lesions and disease transmission. ${ }^{22,23}$ High bacillary titers in cavities increase the probability of establishing drug-resistant bacterial populations. ${ }^{43,44}$ Along with imaging findings, it is therefore understandable that thicker walls and larger cavities are more likely to be associated with MDR-TB and extensively drug-resistant tuberculosis (XDR-TB). ${ }^{45,46}$ While MDR-TBs tend to be more extensive in chest CT imaging than DS-TBs, ${ }^{47}$ imaging signs still cannot independently discriminate MDR-TB from DS-TB. ${ }^{45,48}$ Our findings imply that higher plasma IDO activity indicates more severe MTB destruction in the lung parenchymal of patients, related to more extensive cavity lung lesions (cavity prevalence and cavity size), leading to a considerably higher risk of MDR-TB developing and greater transmission of the pathogen. ${ }^{49,50}$ Although imaging data from more cases is needed to confirm our pilot evaluation in this study, IDO activity may be a feasible tool along with CT imaging for early identification of such patients. This is urgently needed to facilitate promptly conducting the correct TB patients into an isolation ward, thereby alleviating the spread of MDR-TB.

We found significantly higher plasma IDO activity in the MDR-TB group than in the lung cancer group, with a discriminating significance. Several studies have shown that an increase in IDO activity is associated with poor prognosis in cancer patients. ${ }^{51}$ In our study, due to the limitation of a small sample size of lung cancer cases with no small cell lung cancer (NSCLC) cases or small cell lung cancer (SCLC) cases, plasma IDO activity showed no statistical discrimination from HCs. Given the current absence of efficient biomarkers to distinguish MDR-TB from lung cancer (and vice versa), ${ }^{52}$ we call for more feasible and powerful tools for identification of these two diseases. There is overlap between pulmonary tuberculosis and malignant lesions. Using high radiodensities on enhanced CT, MRI or ${ }^{18}$ F-FDG PET/CT is a sensitive tool for identifying lung cancer $^{53,54}$ however especially in MDR-TB patients parenchymal consolidations and scarring with parenchymal and cavitary nodal calcifications are more common and poorly defined, and patchy in reactivated disease, ${ }^{55-57}$ all of which could also be consistent with a lung cancer and thus leading to unnecessary invasive tissue collection and resections in these populations. ${ }^{58}$ An earlier study reported by Seo KJ et al upon analyzing clinical specimens from TB patients, demonstrated that is was possible to use IDO as an immunohistochemical marker to differentiate between tuberculous granuloma (TG) and non-tuberculousgranuloma (NG). ${ }^{23}$ However, the limitations of this method are the invasive procedures required to obtain the pathologic specimens, and the longer duration required for histopathology measurement. IDO's expression in tuberculous granuloma of a nonhuman primate model was found to increase and to be associated with host-Mycobacterium tuberculosis interactions, which either result in acute infection or the control of infection in a latent state. ${ }^{23,40}$ However, we are still uncertain about increasing plasma IDO activity in human extrapulmonary tuberculosis or latent tuberculosis because of the difficulty in collecting human samples with variable confounding factors. ${ }^{40} \mathrm{We}$ assumed that plasma IDO activity in human latent TB or extrapulmonary TB might be increasing but be lower than in human MDR-TB. This assumption will be validated in future study. Recently, another study on serum markers found than the evaluation of circulating cell-free DNA 
(ccfDNA) is more sensitive and specific than carcinoembryonic antigen (CEA), carbohydrate antigen 125 (CA125), and neuron-specific enolase (NSE) in differentiating NSCLC from tuberculosis. ${ }^{59}$ Unfortunately, these approaches are currently costly, with a low throughput. Therefore, our data from a small group of NSCLC patients, while requiring confirmation in future studies with a larger cohort, shed light on the value of plasma IDO activity, which seems to be potentially feasible as a compensatory tool for imaging or histopathology identification of MDR-TB from among other lung malignant diseases. Our pilot study used IDO activity as an auxiliary biomarker to discriminate MDR-TB from DS-TB and lung cancer, which can contribute to developing a combined diagnostic procedure with other biomarkers. For instance, in Chao Liu et al.'s reporting, the panel containing Programmed cell death protein 1 (PD-1), interleukin-10 (IL-10), Interleukin-2 receptor alpha (IL-2R $\alpha$ ) and cancer antigen 15-3 (CA15-3) as biomarkers discriminated breast cancer from benign breast disease with high efficiency. ${ }^{60}$

Several limitations exist in this study. The sample cases were selected within the restrictions of distance, transport duration, and parallel period $(2 \mathrm{~h})$ for blood drawing to keep the temperature and biological metabolism conditions of the body consistent (the present cases were all from east China). Thus, future studies should be performed with more cases of MDR-TB from different regions, along with other pulmonary infections and more malignant cavitary lung disease cases as controls to determine if increasing of IDO activity is specific to MDR-TB. Second, we examined IDO activity, determined by the plasma Kyn/Trp ratio, but lacked molecular or pathological confirmation of IDO expression in these patients. Origin of increased IDO activity in the plasma of MDR-TB patients in this study is still not clear in terms of clarifying the mechanisms in MDR-TB. Previous studies have focused mostly on TB conditions, with uncertainty around MDR-TB. Future studies will be required to clarify these issues. Third, further studies evaluating MDR-TB patients from baseline to after a standard treatment regimen, at different time points, should be performed to confirm that IDO activity could be used to assist diagnosis of MDR-TB and to monitor anti-TB therapy.

\section{Conclusion}

IDO activity in MDR-TB patients was predominantly higher than that in DS-TB patients and HCs, with the diagnostic significance in discriminating from the DSTB group and lung cancer group. We found that IDO activity was positively correlated with cavitary lung lesions in patients with $\mathrm{TB}$, potentially indicating a more severe infectious condition and higher risk of developing MDR-TB. We conducted this study to propose and test plasma IDO activity as a novel biomarker candidate for the early detection of MDR-TB from DSTB. Our finding suggested that plasma IDO activity can be used as a potential biomarker to assist chest CT scans in the early detection of MDR-TB. However, this study is only a pilot study, and a large-scale study is needed to confirm our findings. This pilot study allows a better understanding of IDO's participation in the pathogenesis of MDR- TB and DS - TB, and can contribute to developing a combined diagnostic procedure with other biomarkers.

\section{Acknowledgments}

The authors would like to thank Fu-Qiang Wang and Analysis Center Nanjing Medical University for their comments and assistance in high performance liquid chromatography-mass spectrometry measurement and blood sample preparation.

This work was funded by The Key Project of National Science \& Technology for Infectious Diseases of China [Grants \#2015ZX10003001], [Grants \#2018ZX10722301]; National Natural Science Foundation of China [Grants \#81800011]; The Key Research and Development Project of Zhenjiang, China [Grants \#SH2017022]

\section{Disclosure}

The authors report no conflicts of interest in this work.

\section{References}

1. Van der Heijden YF, Abdullah F, Andrade BB, et al. Building capacity for advances in tuberculosis research; proceedings of the third report international meeting. Tuberculosis (Edinb). 2018;113:153-162. doi:10.1016/j.tube.2018.09.009

2. World Health Organization. Global tuberculosis report 2018, Geneva. https://www.who.int/tb/publications/global_report/en/. Accessed September 18, 2018.

3. World Health Organization (Producer). Use of high burden country lists for TB by WHO in the post-2015 era, June 2015 meeting of WHO's Strategic and technical advisory group for TB (STAG-TB). WHO/HTM/TB/2015.29. Geneva: World Health Organization; 2015

4. Andrews JR, Noubary F, Walensky RP, Cerda R, Losina E, Horsburgh CR. Risk of progression to active tuberculosis following reinfection with Mycobacterium tuberculosis. Clin Infect Dis Mar. 2012;54(6):784-791. 
5. Shah NS, Auld SC, Brust JC, et al. Transmission of extensively drug-resistant tuberculosis in South Africa. $N$ Engl J Med. 2017;376(3):243-253.

6. Dowdy DW, Azman AS, Kendall EA, Mathema B. Transforming the fight against tuberculosis: targeting catalysts of transmission. Clin Infect Dis. 2014;59(8):1123-1129.

7. World Health Organization. WHO Treatment Guidelines for DrugResistant Tuberculosis. Geneva: World Health Organization; 2016.

8. Virenfeldt J, Rudolf F, Camara C, et al. Treatment delay affects clinical severity of tuberculosis: a longitudinal cohort study. $B M J$ Open. 2014;4(6):e004818.

9. Zumla A, George A, Sharma V, Herbert RH, Oxley A, Oliver M. The WHO 2014 global tuberculosis report-further to go. Lancet Glob Health. 2014;3(1):e10-e12.

10. Boehme CC, Nabeta P, Hillemann D, et al. Rapid molecular detection of tuberculosis and rifampin resistance. $N$ Engl J Med. 2010;363 (11):1005-1015. doi:10.1056/NEJMoa0907847

11. Uplekar M, Weil D, Lonnroth K, et al. WHO's new end TB strategy. Lancet. 2015;385(9979):1799-1801. doi:10.1016/S0140-6736(15)60570-0

12. Lawn SD, Mwaba P, Bates M, et al. Advances in tuberculosis diagnostics: the Xpert MTB/RIF assay and future prospects for a point-of-care test. Lancet Infect Dis. 2013;13(4):349-361. doi:10.1016/S14733099(13)70008-2

13. Dorman SE, Schumacher SG, Alland D, et al. Xpert MTB/RIF ultra for detection of Mycobacterium tuberculosis and rifampicin resistance: a prospective multicentre diagnostic accuracy study. Lancet Infect Dis. 2018;18(1):76-84. doi:10.1016/S1473-3099(17)30691-6

14. Gupta S, Kakkar V. Recent technological advancements in tuberculosis diagnostics - A review. Biosens Bioelectron. 2018;115:14-29. doi:10.1016/j.bios.2018.05.017

15. UNITAID. Tuberculosis: Diagnostics Technology Landscape. 5th. Geneva, Switzerland; 2017.

16. Wallis RS, Kim P, Cole S, et al. Tuberculosis biomarkers discovery: developments, needs, and challenges. Lancet Infect Dis. 2013;13 (4):362-372. doi:10.1016/S1473-3099(13)70034-3

17. Pfefferkorn ER. Interferon gamma blocks the growth of Toxoplasma gondii in human fibroblasts by inducing the host cells to degrade tryptophan. Proc Natl Acad Sci U S A. 1984;81(3):908-912.

18. Taylor MW, Feng GS. Relationship between interferon-gamma, indoleamine 2,3-dioxygenase, and tryptophan catabolism. FASEB J. 1991;5(11):2516-2522.

19. Werner ER, Bitterlich G, Fuchs D, et al. Human macrophages degrade tryptophan upon induction by interferon-gamma. Life Sci. 1987;41(3):273-280.

20. Carlin JM, Borden EC, Sondel PM, Byrne GI. Interferon-induced indoleamine 2,3-dioxygenase activity in human mononuclear phagocytes. J Leukoc Biol. 1989;45(1):29-34.

21. Curti A, Trabanelli S, Salvestrini V, Baccarani M, Lemoli RM. The role of indoleamine 2,3-dioxygenase in the induction of immune tolerance: focus on hematology. Blood. 2009;113(11):2394-2401. doi:10.1182/blood-2008-07-144485

22. Gautam US, Foreman TW, Bucsan AN, et al. In vivo inhibition of tryptophan catabolism reorganizes the tuberculoma and augments immune-mediated control of Mycobacterium tuberculosis. Proc Natl Acad Sci U S A. 2018;115(1):E62-E71. doi:10.1073/pnas.1711373114

23. Seo KJ, Yoo CY, Im SY, et al. A possible complementary tool for diagnosing tuberculosis: a feasibility test of immunohistochemical markers. Int J Clin Exp Pathol. 2015;8(11):13900-13910.

24. Suzuki Y, Suda T, Asada K, et al. Serum indoleamine 2,3-dioxygenase activity predicts prognosis of pulmonary tuberculosis. Clin Vaccine Immunol. 2012;19(3):436-442. doi:10.1128/CVI.05402-11

25. Suzuki Y, Miwa S, Akamatsu T, et al. Indoleamine 2,3-dioxygenase in the pathogenesis of tuberculous pleurisy. Int $J$ Tuberc Lung Dis. 2013;17(11):1501-1506. doi:10.5588/ijtld.13.0082
26. Van Laarhoven A, Dian S, Aguirre-Gamboa R, et al. Cerebral tryptophan metabolism and outcome of tuberculous meningitis: an observational cohort study. Lancet Infect Dis. 2018;18(5):526-535. doi:10.1016/S1473-3099(18)30053-7

27. Barry S, Breen R, Lipman M, Johnson M, Janossy G. Impaired antigen-specific $\mathrm{CD} 4(+) \mathrm{T}$ lymphocyte responses in cavitary tuberculosis. Tuberculosis (Edinb). 2009;89(1):48-53. doi:10.1016/j. tube.2008.07.002

28. Tan Q, Xie WP, Min R, et al. Characterization of Th1- and Th2-type immune response in human multidrug-resistant tuberculosis. Eur J Clin Microbiol Infect Dis. 2012;31(6):1233-1242. doi:10.1007/ s10096-011-1434-4

29. Tan Q, Min R, Wang H, et al. Modulation of IFN-gamma and IL-17 is critical in human immune responses to multidrug-resistant tuberculosis in Eastern Chinese Han population. Respirology Supplement, 16th Congress of the Asian Pacific Society of Respirology, Shanghai, China, 05 November 2011, APSR-ONL-885,2: 16.

30. Russell DG, Barry CE 3rd, Flynn JL. Tuberculosis: what we don't know can, and does, hurt us. Science. 2010;328(5980):852-856. doi: $10.1126 /$ science. 1184784

31. Multidrug-resistant tuberculosis (MDR-TB) indicators. A minimum set of indicators for the programmatic management of MDR-TB in national tuberculosis control programmes. WHO/HTM/TB/2010.11. Geneva: World Health Organization; 2010

32. Falzon D, Mirzayev F, Wares F, et al. Multidrug-resistant tuberculosis around the world: what progress has been made? Eur Respir J. 2015;45(1):150-160. doi:10.1183/09031936.00101814

33. Zhang YJ, Reddy MC, Ioerger TR, et al. Tryptophan biosynthesis protects mycobacteria from CD4 T-cell-mediated killing. Cell. 2013;155(6):1296-1308. doi:10.1016/j.cell.2013.10.045

34. Cervenka I, Agudelo LZ, Ruas JL. Kynurenines: tryptophan's metabolites in exercise, inflammation, and mental health. Science. 2017;357(6349):eaaf9794. doi:10.1126/science.aaf9794

35. Mellor AL, Keskin DB, Johnson T, Chandler P, Munn DH. Cells expressing indoleamine 2,3-dioxygenase inhibit $\mathrm{T}$ cell responses. J Immunol. 2002;168(8):3771-3776.

36. Fallarino F, Grohmann U, Vacca C, et al. T cell apoptosis by kynurenines. Adv Exp Med Biol. 2003;527:183-190.

37. Fallarino F, Grohmann U, You S, et al. The combined effects of tryptophan starvation and tryptophan catabolites down-regulate $\mathrm{T}$ cell receptor zeta-chain and induce a regulatory phenotype in naive T cells. J Immunol. 2006;176(11):6752-6761.

38. Munn DH, Sharma MD, Lee JR, et al. Potential regulatory function of human dendritic cells expressing indoleamine 2,3-dioxygenase. Science. 2002;297(5588):1867-1870.

39. Munn DH, Zhou M, Attwood JT, et al. Prevention of allogeneic fetal rejection by tryptophan catabolism. Science. 1998;281 (5380):1191-1193.

40. Mehra S, Alvarez X, Didier PJ, et al. Granuloma correlates of protection against tuberculosis and mechanisms of immune modulation by Mycobacterium tuberculosis. J Infect Dis. 2013;207 (7):1115-1127

41. Li N, Xie WP, Kong H, et al. Enrichment of regulatory T-cells in blood of patients with multidrug-resistant tuberculosis. Int J Tuberc Lung Dis. 2015;19(10):1230-1238.

42. Adu-Gyamfi CG, Snyman T, Hoffmann CJ, et al. Plasma indoleamine 2, 3-dioxygenase, a biomarker for tuberculosis in human immunodeficiency virus-infected Patients. Clin Infect Dis. 2017;65(8):1356-1358.

43. Mazzarella G, Bianco A, Perna F, et al. T lymphocyte phenotypic profile in lung segments affected by cavitary and non-cavitary tuberculosis. Clin Exp Immunol. 2003;132(2):283-288.

44. Ong CW, Elkington PT, Friedland JS. Tuberculosis, pulmonary cavitation, and matrix metalloproteinases. Am J Respir Crit Care Med. 2014;190(1):9-18. 
45. Cha J, Lee HY, Lee KS, et al. Radiological findings of extensively drug-resistant pulmonary tuberculosis in non-AIDS adults: comparisons with findings of multidrug-resistant and drug-sensitive tuberculosis. Korean J Radiol. 2009;10(3):207-216.

46. Cheon H. Comparison of CT findings of between MDR-TB and XDR-TB: a propensity score matching study. Imaging Med. 2017;9:125-129.

47. Fishman JE, Sais GJ, Schwartz DS, Otten J. Radiographic findings and patterns in multidrug-resistant tuberculosis. J Thorac Imaging. 1998;13(1):65-71.

48. Kim SH, Min JH, Lee JY. Radiological findings of primary multidrug-resistant pulmonary tuberculosis in HIV-seronegative patients. Hong Kong J Radiol. 2014;17:4-8.

49. Van Dyck P, Vanhoenacker FM, Van Den Brande P, De Schepper AM. Imaging of pulmonary tuberculosis. Eur Radiol. 2003;13(8):1771-1785.

50. Zhang L, Pang Y, Yu X, et al. Risk factors for pulmonary cavitation in tuberculosis patients from China. Emerg Microbes Infect. 2016;5 (10):e110.

51. Platten M, Wick W. Van den Eynde BJ. Tryptophan catabolism in cancer: beyond IDO and tryptophan depletion. Cancer Res. 2012;72 (21):5435-5440.

52. Ho JC, Leung CC. Management of co-existent tuberculosis and lung cancer. Lung Cancer. 2018;122:83-87.

53. Qi LP, Chen KN, Zhou XJ, et al. Conventional MRI to detect the differences between mass-like tuberculosis and lung cancer. $J$ Thorac Dis. 2018;10(10):5673-5684.
54. Niyonkuru A, Bakari KH, Lan X. (18)F-fluoro-2-deoxy-d-glucose PET/computed tomography evaluation of lung cancer in populations with high prevalence of tuberculosis and other granulomatous disease. PET Clin. 2018;13(1):19-31.

55. Lang S, Sun J, Wang X, et al. Asymptomatic pulmonary tuberculosis mimicking lung cancer on imaging: a retrospective study. Exp Ther Med. 2017;14(3):2180-2188.

56. Kim YI, Goo JM, Kim HY, Song JW, Im JG. Coexisting bronchogenic carcinoma and pulmonary tuberculosis in the same lobe: radiologic findings and clinical significance. Korean J Radiol. 2001;2 (3):138-144.

57. Yu YY, Pinsky PF, Caporaso NE, et al. Lung cancer risk following detection of pulmonary scarring by chest radiography in the prostate, lung, colorectal, and ovarian cancer screening trial. Arch Intern Med. 2008;168(21):2326-2332. discussion 2332.

58. Davies PD, Pai M. The diagnosis and misdiagnosis of tuberculosis. Int J Tuberc Lung Dis. 2008;12(11):1226-1234.

59. Leng S, Zheng J, Jin Y, et al. Plasma cell-free DNA level and its integrity as biomarkers to distinguish non-small cell lung cancer from tuberculosis. Clin Chim Acta. 2018;477:160-165.

60. Liu C, Sun B, Xu B, et al. A panel containing PD-1, IL-2Ralpha, IL-10, and CA15-3 as a biomarker to discriminate breast cancer from benign breast disease. Cancer Manag Res. 2018;10:1749-1761.
Infection and Drug Resistance

\section{Publish your work in this journal}

Infection and Drug Resistance is an international, peer-reviewed openaccess journal that focuses on the optimal treatment of infection (bacterial, fungal and viral) and the development and institution of preventive strategies to minimize the development and spread of resistance. The journal is specifically concerned with the epidemiology of

\section{Dovepress}

antibiotic resistance and the mechanisms of resistance development and diffusion in both hospitals and the community. The manuscript management system is completely online and includes a very quick and fair peerreview system, which is all easy to use. Visit http://www.dovepress.com/ testimonials.php to read real quotes from published authors. 